\title{
Low-energy constants in relativistic chiral nucleon-nucleon potential
}

\author{
Renato Higa \\ Jefferson Lab, 12000 Jefferson Ave, Newport News, VA 23606, USA
}

\begin{abstract}
In this work we focus on the possibility of extract low energy constants via fits from phase shifts of nucleon-nucleon scattering, pointing out the differences between the relativistic and heavy baryon formulations of chiral perturbation theory. Preliminary results indicate that these two formulations give slightly different predictions for phase shifts, affecting the extraction of the low energy constants.
\end{abstract}

\section{INTRODUCTION}

After more than fifty years of intense research it became well established that this basic theory, QCD, can be represented by means of chiral perturbation theory $(\mathrm{ChPT})$ in the low-energy region. In the phase diagram of QCD this corresponds to small values of temperature and density, where QCD is non-perturbative. Even though it covers only a limited area of the phase diagram, ChPT can be used as a good guide for extrapolations to intermediate energies still inaccessible by perturbative QCD. The chiral lagrangian is described in terms of low energy constants (LECs). In processes involving baryons, they are often determined through pion-nucleon processes, but the existing values come with large uncertainties [1]. They are also present in the nucleon-nucleon $(N N)[3,4,5]$ and few nucleon [6] systems constructed via ChPT, with reasonable description of scattering observables given as predictions. With the large amount of $N N$ scattering data available [7] it is interesting to, conversely, use of them to fix some of these LECs. This idea was first proposed by the Nijmegen group [8], who claims to have extracted three of them with an accuracy of 2-5\%. Apart from the fact that their procedure might not be reliable (see [9] for instance) there is also the discussion about whether the heavy baryon formalism employed is apropriate for these purposes. In the following sections we bring this latter question into consideration, showing how they can possibly interfere the determination of LECs.

\section{RELATIVISTIC VERSUS HEAVY BARYON}

Contrary to what happens in ChPT in the mesonic sector, with baryons the theory gets more complicated. The first and widely used solution is to apply a sort of non-relativistic expansion, the so called heavy baryon expansion. Even though most of the chiral $N N$ potentials are based on these ideas, there are problems regarding the validity of such an 


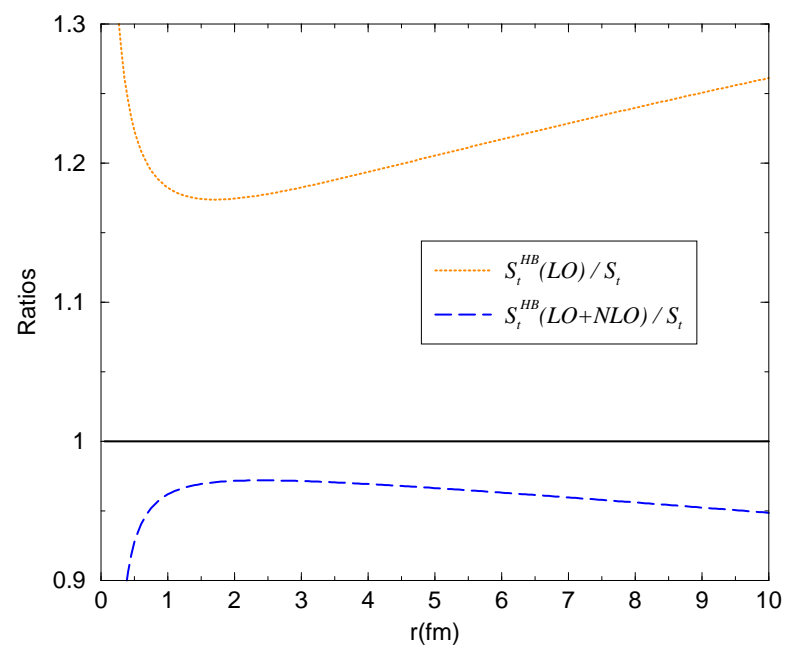

FIGURE 1. Ratios of the heavy baryon expansion for the triangle diagram by its relativistic version.

expansion, as pointed out by Becher and Leutwyler [10]. Relativistic approaches which avoid these problems where proposed [10] and applied to the $N N$ system [4, 5]. An important result was shown in [5], that the heavy baryon formalism fails to describe the asymptotic behavior of the $N N$ potential. This is ilustrated in fig.1, where a basic relativistic loop integral that describes the potential (the "triangle" diagram, denoted by $S_{t}$ ) is expanded in powers of $1 / M$, where $M$ is the baryon mass. In the heavy baryon potential up to $O\left(q^{4}\right)$ this integral contributes up to next-to-leading order (NLO), but in some cases (for instance, where this integral is multiplied by $m_{\pi}^{3}$ ) only the first term is kept. Clearly this is a crude approximation, and seems to diverge for large distances.

\section{LECS AND PHASE SHIFTS}

As mentioned before, the LECs are present not only in pion-nucleon, but also in fewbody nuclear physics, the most important being $c_{1}, c_{3}$ and $c_{4}$. The accepted values for them lies in the following range [6],

$$
c_{1}=-0.81 \cdots-1.53, \quad c_{3}=-4.70 \cdots-6.19, \quad c_{4}=3.25 \cdots 4.12 .
$$

Such values allow variations of almost two times the strengh of the central isoscalar potential (by far, the most important component for the $N N$ phenomenology) in the region between 1.4 and $5 \mathrm{fm}$. The Nijmegen values, designated here as set 1 , are $c_{1}=-0.76(7), c_{3}=-4.78(10)$, and $c_{4}=3.96(22)$. In order to see the variation of phase shifts on these parameters, we use the values from Büttiker and Meißner [1] (set 2): $c_{1}=-0.81, c_{3}=-4.7$, and $c_{4}=3.25$.

In fig. 2 we show the dependence of the ${ }^{3} F_{3}$ phase shift on the values of the LECs used, as well as the formalism used to obtain the potential. This clearly ilustrates that the values for the LECs extracted from phase shifts depends on which formalism one is working with. Due to the odds of the heavy baryon framework to describe the long 


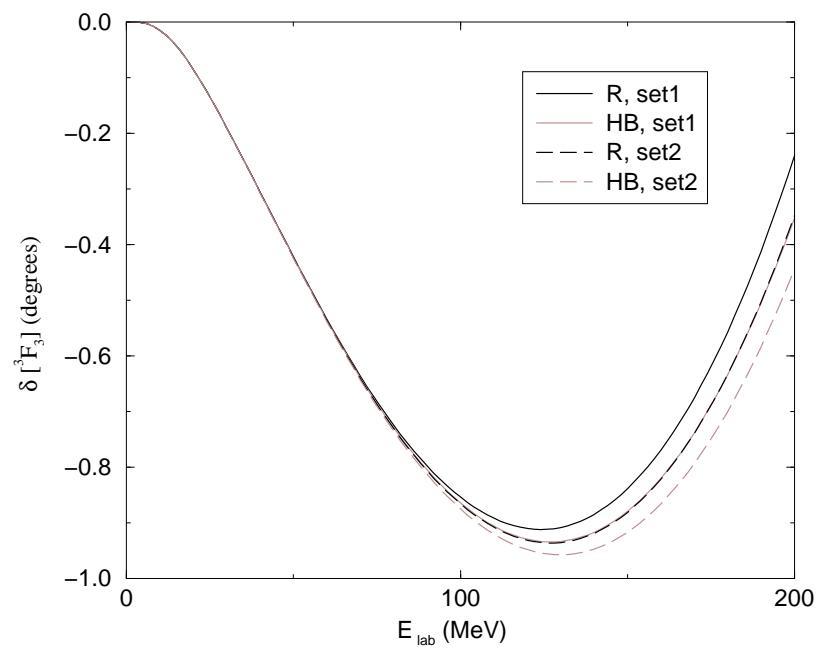

FIGURE 2. ${ }^{3} F_{3}$ phase shift, as a function of energy. The black (gray) curves correspond to the relativistic (heavy baryon) potential, calculated using the LECs from Refs. [8] (set 1) and [1] (set 2).

distance properties of the $N N$ interaction, we argue here that, for a determination of LECs from phase shifts with a well grounded theoretical basis is necessary to use the relativistic potential.

\section{ACKNOWLEDGMENTS}

I would like to thank the hospitality of the Institute for Nuclear Theory at the University of Washington, where part of this work was developed, and the organizers of Hadron/RANP 2004, for the oportunity of join this pleasant meeting. This work was supported by DOE Contract No.DE-AC05-84ER40150 under which SURA operates the Thomas Jefferson National Accelerator Facility.

\section{REFERENCES}

1. P. Büttiker and U.-G. Meißner, Nucl. Phys. A668, 97 (2000).

2.

3. C. Ordóñez, L. Ray, and U. van Kolck, Phys. Rev. Lett. 72, 1982 (1994); N. Kaiser, R. Brockman, and W. Weise, Nucl. Phys. A625, 758 (1997); D. R. Entem and R. Machleidt, Phys. Rev. C 66, 014002 (2002); E. Epelbaum, W. Glöckle, and U-G. Meißner, Eur. Phys. J. A 19, 125 (2004).

4. R. Higa and M.R. Robilotta, Phys. Rev. C 68, 024004 (2003).

5. R. Higa, M. R. Robilotta e C. A. da Rocha, Phys. Rev. C 69, 034009 (2004).

6. E. Epelbaum, A. Nogga, W. Glöckle, H. Kamada, U.-G. Meißner, and H. Witala, Eur. Phys. J. A 15 (2002), 543.

7. V. G.J. Stoks, R. A. M. Klomp, M. C. M. Rentmeester, and J. J. de Swart, Phys. Rev. C 48, 792 (1993); R. Arndt, I. I. Strakovsky, and R. L. Workman, Phys. Rev. C 50, 2731 (1994).

8. M. C. M. Rentmeester, R. G. E. Timmermans, and J. J. de Swart, Phys. Rev. C 67, 044001 (2003).

9. D.R. Entem and R. Machleidt, preprint nucl-th/0303017 (2003).

10. T. Becher and H. Leutwyler, Eur. Phys. J. C 9, 643 (1999); J. High Energy Phys. 106, 17 (2001). 